Proceedings of the 17th Czech and Slovak Conference on Magnetism, Košice, Slovakia, June 3-7, 2019

\title{
Monitoring of Rail Surface Damage via Magnetic Measurements
}

\author{
K. ZGÚtová ${ }^{a, *}$, M. NeSlušan ${ }^{a}$, P. MinÁRIK ${ }^{b}$ AND J. ŠrŔ́MEK ${ }^{a}$ \\ ${ }^{a}$ University of Žilina, Univerzitná 1, 01026 Žilina, Slovakia \\ ${ }^{b}$ Charles University, Ke Karlovu 5, 12116 Praha 2, Czech Republic
}

\begin{abstract}
This paper reports about magnetic non-destructive evaluation of the rail surface exposed to long-time cyclic plastic deformation. Long-term severe plastic deformation and superimposing friction heating initiate pearlite matrix defragmentation and the corresponding refinement of magnetic domain structure. This paper discusses potential of the Barkhausen noise as well as tangential magnetic field techniques for such purpose. The paper analyses correlation between the thickness of the near surface white layer and magnetic parameters. This study would contribute to a possible concept for preventing unexpected rails deformation (or cracking) due to their thermal dilatation via non-destructive magnetic techniques.
\end{abstract}

DOI: 10.12693/APhysPolA.137.644

PACS/topics: rail surface, Barkhausen noise, tangential magnetic field

\section{Introduction}

Large axle loads of the trains usually cause serious wear and structure transformations of the rails. White etching layer (WEL) is a typical defect in the surface region of the rail originating from repetitive and severe plastic deformation [1]. WEL is a generic term for very hard surface layer which appears white on micrographs due to high resistance against etching. It was reported that WEL in the surface of rails is inhomogeneous and composed of several different microstructures [2]. WEL as a hard structure may lead to the brittle cracking and subsequent fatigue crack propagation. It was reported [1] that WEL is a product of rolling-contact fatigue caused by the alternating stresses associated with rolling contact bodies. Operation of the rails containing WEL regions might be risky due to possible macrocracking initiated by microcracks in the WEL. Furthermore, rails are subjected to grinding process in order to remove damaged layer and surface asperities [3]. For these reasons, a suitable nondestructive method would be beneficial for assessment of the degree of rail surface damage. Magnetic techniques such as the Barkhausen noise (MBN) or/and distortion of tangential magnetic field are sensitive to microstructure and stress state. This study deals with their sensitivity for such purpose (as the promising technique for monitoring rails surface).

\section{Experiments}

The experimental study was carried out on hot rolled pearlite rail steel R220 (strength $785 \pm 50 \mathrm{MPa}$, hardness $215 \pm 35 \mathrm{HB}, 0.6 \% \mathrm{C}, 1.05 \% \mathrm{Mn}, 0.21 \% \mathrm{Si}$, and $0.12 \% \mathrm{Cr}$ ).

\footnotetext{
* corresponding author; e-mail:

katarina.zgutova@fstav.uniza.sk
}

The rail was subjected to passing tonnage accumulated over 20 years, with about 0.8 million tons per year. MBN was measured by the use of RollScan 350 (magnetizing voltage $5 \mathrm{~V}$, magnetizing frequencies $30-425 \mathrm{~Hz}$, frequency range of MBN $70-200 \mathrm{kHz}$ ). MBN refers to the root-mean-square (rms, effective) value of the signal. Harmonic analysis of the tangential magnetic field strength (TMFS) was measured by the use 3MA II device and sensor 3MA WPM/HF S15226 (sine profile of excitation magnetic field of amplitude $50 \mathrm{~A} \mathrm{~cm}^{-1}$ and frequency of $125 \mathrm{~Hz}$ ). $10 \mathrm{~mm}$ long pieces were routinely prepared for metallographic and scanning electron microscopy (SEM) observations (etched by $3 \%$ Nital for $8 \mathrm{~s}$ ) to reveal the microstructure transformations induced by severe plastic deformation. All measurements were conducted at 7 points within the rail surface. The first position was placed $3 \mathrm{~mm}$ from the left side. The next positions were spaced by $6 \mathrm{~mm}$ from each other. Distribution of magnetic domains was investigated by atomic force microscope (AFM) Bruker Dimension EDGE in the MFM mode. Samples for the measurement were mechanically polished down to $50 \mathrm{~nm}$ diamond paste.

\section{Results and their discussions}

Figure 1 shows micrographs of the rail surface containing WEL. This layer can be easily contrasted against the deeper pearlite regions appearing dark. Figures 1 and 2 also show that WEL thickness is asymmetrical with respect to the rail width. The maxima of WEL thickness can be found on the left side of the rail (especially positions at 9 and $15 \mathrm{~mm}$ ), whereas other positions remain less affected. Such asymmetry is due to the contact geometry in the wheel-rail contact due to certain tilting of the rail during the wheel-rail interaction [4]. The main interaction between the rails and wheel surfaces is therefore near the wheel flange, which in turn corresponds to the inner part of the rails. 

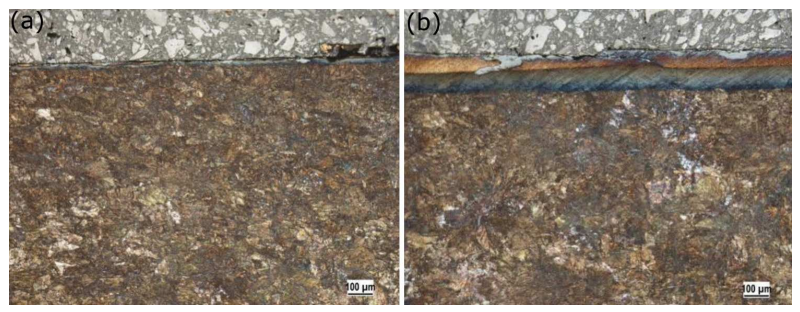

Fig. 1. Metallographic images of the rail surface: (a) distance $3 \mathrm{~mm}$ from the left side, (b) distance $15 \mathrm{~mm}$ from the left side.

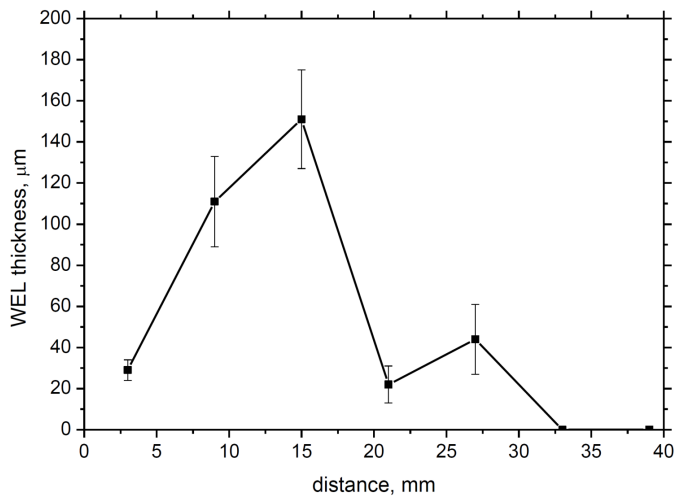

Fig. 2. Distribution of WEL thickness.

Figure 3 demonstrates that $\mathrm{MBN}$ is sensitive to the deeper extent of surface damage and $\mathrm{MBN}$ is inversely proportional to the thickness of WEL. It was found that WEL has the martensite structure formed after austenitisation due to rise in temperature caused by frictional heating in the rail-wheel contact areas [1]. As it was discussed in the previous study [5], remarkably lower MBN in the thick WEL are due to martensite matrix of high dislocation density, austenite, and oxides in the WEL as the pinning sites hinder domain wall motion. Figure 3 also shows that better sensitivity of MBN signal against WEL thickness can be found for magnetizing frequency of $125 \mathrm{~Hz}$ and more. Such finding is driven by domain wall dynamics, especially $\mathrm{d} H / \mathrm{d} t$ rate during cycling magnetization. However, Fig. 3 also depicts that influence of magnetizing frequency saturates early and sensitivity of MBN against WEL thickness above the magnetizing frequency $125 \mathrm{~Hz}$ is nearly the same.

It was found [5] that the initial pearlite lamellas are completely fragmented. On the one side, such fragmentation occurs together with structure alterations (martensite, oxides, austenite, etc.). On the other hand, Fig. 4 illustrates that matrix defragmentation results in refinement of domain configuration. Long-term severe plastic deformation at elevated temperatures makes domain structure finer which in turns increases domain wall density. Consequently, the number of MBN jumps and thus MBN activity would increase. However, the decreased MBN along with decreased grain size is usually associated with severe plastic deformation [6]. MBN as the

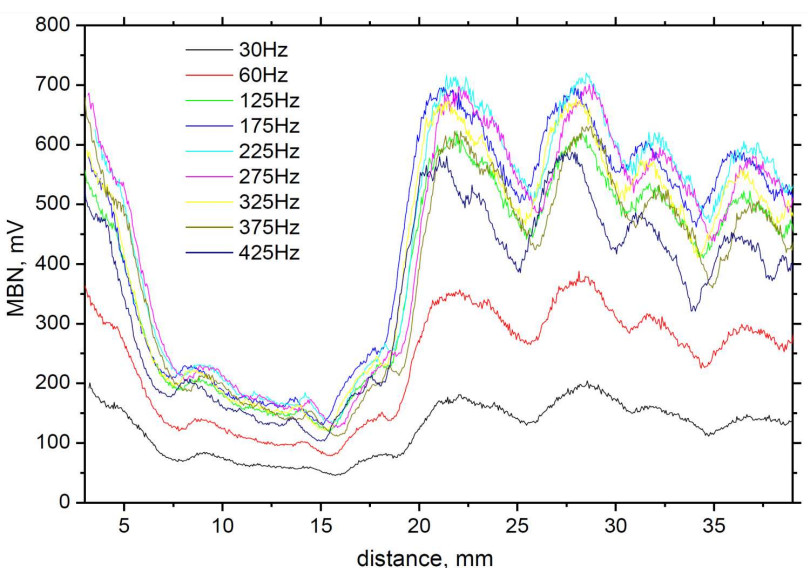

Fig. 3. Dynamic records of MBN within the rail width, variable magnetizing frequencies.
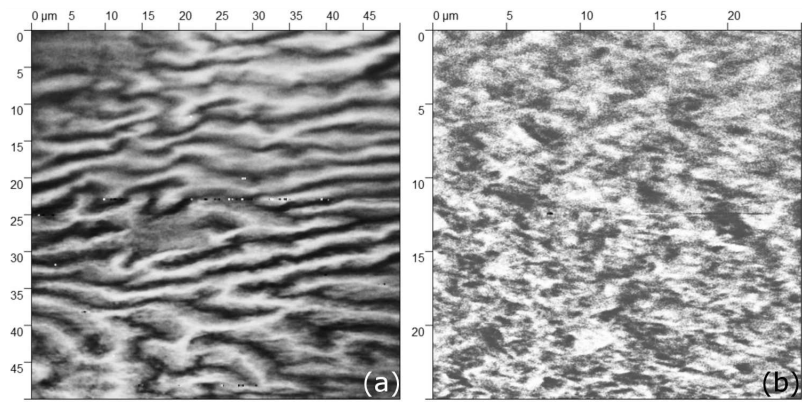

Fig. 4. Magnetic domain structure, MFM technique: (a) bulk magnetic domain structure, (b) WEL magnetic domain structure.

effective value is a function of MBN pulses and their amplitude. Number of MBN pulses for magnetizing frequency of $30 \mathrm{~Hz}$ is approximately 147000 (in this particular case) and decreases with magnetizing frequency up to 7600 pulses for frequency of $425 \mathrm{~Hz}$.

Figures 2, 3, and 4 illustrate that the effect of decreasing amplitude of MBN pulses dominates over their increasing frequency. Furthermore, MBN jumps at higher magnetizing frequencies occur in the form of avalanches and measuring system is not capable to distinguish among the individual MBN pulses.

MBN technique usually requires cyclic magnetisation of a sample by the alternating magnetic field. This field is carried out by a sinusoidal excitation with the yoke. 3MA II system contains the Hall probe sitting in the middle between the pole shoes which measures the time signal of the tangential magnetic field in the sample. It can be found that the measured magnetic field is not sinusoidal. Distortions are clearly visible, especially in the range after the zero transition. The reason for this is the nonlinear behaviour of the ferromagnetic hysteresis [7, 8]. By fast Fourier analysis (FFT) of the measured field a fundamental wave and the upper harmonic parts can be numerically determined 


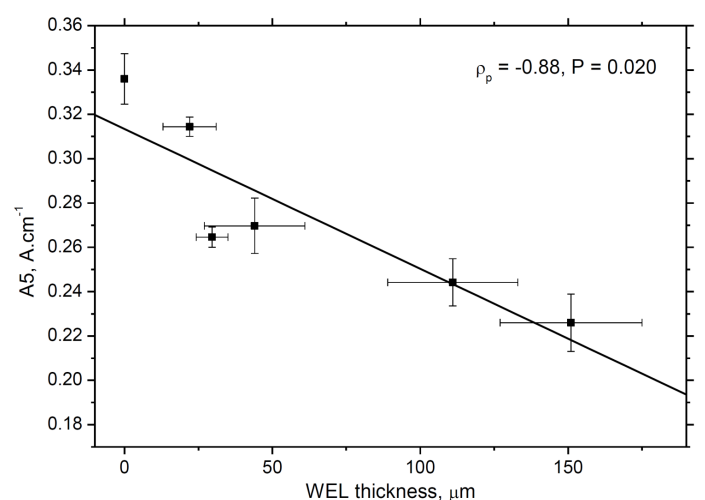

Fig. 5. WEL thickness versus A5 (extracted from TMFS).

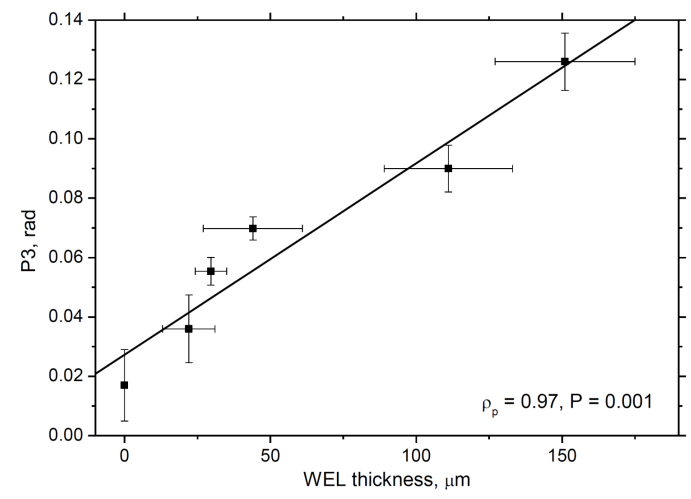

Fig. 6. WEL thickness versus P3 (extracted from TMFS).

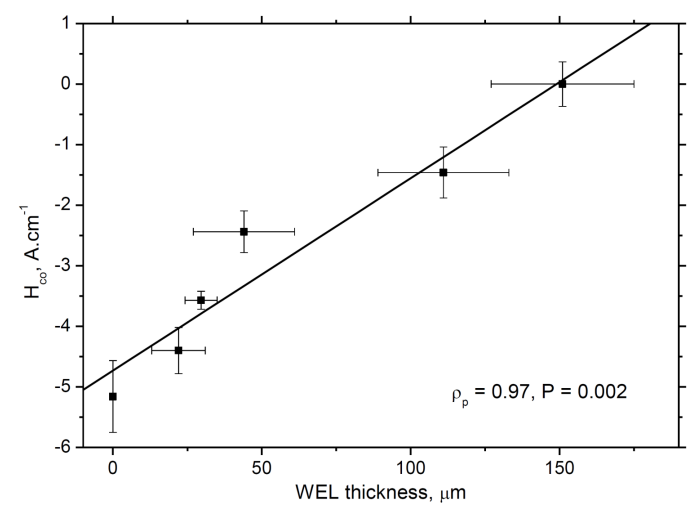

Fig. 7. WEL thickness versus $\mathrm{H}_{\mathrm{co}}$ (extracted from TMFS).

(A3, A5, and A7 as the amplitudes of 3rd, 5th, and 7th harmonics of measured TMFS; P3, P5, and P7 as the phase shifts of $3 \mathrm{rd}, 5 \mathrm{th}$, and 7 th harmonics compared to the fundamental wave; $H_{\mathrm{co}}$ as the coercive field strength derived from measured TMFS, and finally $K$ as the harmonic distortion defined by Eq. (1) [7]). These upper harmonic parts can give information about ferromagnetic properties of the tested material [7, 8].
For instance, it was found that $H_{\mathrm{co}}$ correlates well with coercivity determined from hysteresis measurements [8].

$$
\left.K=\sqrt{(} A_{3}^{2}+A_{5}^{2}+A_{7}^{2}\right) / A_{1}^{2}[\%] .
$$

Figures 5-7 demonstrate that upper harmonic parts (except parameters $A_{3}$ and $K$ ) correlate very well with WEL thickness. Therefore the magnetic technique based on the distortion of TMFS can be employed for assessment of WEL thickness in the rail surface. Especially, all phase shift components $P$ and $H_{\text {co }}$ exhibit good sensitivity against WEL thickness and very high correlation coefficients $\rho_{p}$.

\section{Conclusions}

Microcracking in the hard and brittle WEL arises from the cyclic long term loading and potentially can initiate macrocracking in the rail. For this reason, rails should be subjected to the re-grinding process that removes the damaged layer. Magnetic techniques could be potentially employed for assessment of WEL thickness and therefore this paper suggests the possible concepts to prevent the unexpected rail failures. The possible practical application of MBN technique can be carried out with the static measurements in certain positions with respect to rail width and length. More sophisticated and automated system could employ the dynamic measurement when a rail surface is scanned continuously by the use of multiple sensors placed at different positions with respect to rail width.

\section{Acknowledgments}

This work was supported by the VEGA projects no. $1 / 0170 / 17$ and no. $1 / 0121 / 17$.

\section{References}

[1] S. Pal, W.J.T. Daniel, C.H.G. Valente, A. Wilson, A. Atrens, Eng. Fail. Anal. 22, 152 (2012).

[2] J. Takahashi, K. Kawakami, M. Ueda, Acta Mater. 58, 3602 (2010).

[3] J.F. Santa, A. Toro, R. Lewis, Tribo. Int. 95, 5 (2016).

[4] Y. Ma, V. Markine, A.A. Mashal, M. Ren, Tribol. Int. 114, 264 (2017).

[5] M. Neslušan, J. Č́žžek, K. Zgútová, P. Kejzlar, J. Šrámek, J. Čapek, P. Hruška, O. Melikhova, Wear 402-403, 38 (2018).

[6] M. Neslušan, P. Minárik, J. Grenčík, K. Trojan, K. Zgútová, Wear 420-421, 195 (2019).

[7] 3MA II Training manual, Fraunhofer IZFP, Saarbrucken (Germany) 2013.

[8] G. Dobmann, H. Pitsch, in: Proc. Nondestructive Evaluation of Materials, Saarbrucken (Germany), 1989, p. 636. 